\title{
Zur Theorie der absoluten Thermokraft dünner Metallschichten und -drähte
}

\author{
Von E. Justi, M. Kohler und G. Lautz
}

Aus den Instituten für technische und theoretische Physik der Techn. Hochschule Braunschweig

(Z. Naturforschg. 6 a, 544-550 [1951]; eingegangen am 27. August 1951)

Im Rahmen der Elektronentheorie der Metalle wird die absolute Thermokraft dünner Metallschichten und -drähte berechnet und mit eigenen Messungen an aufgedampften Blei- und Wismutschichten verglichen. Theoretisch kann gegenüber dem kompakten Material nur dann eine Änclerung auftreten, wenn die mittlere freie Weglänge der Elektronen energieabhängig ist. Damit kann man aus derartigen Thermokraftmessungen tiefere Aussagen über den Leitungsmechanismus der Versuchsproben gewinnen als aus dem Schichtdickeneffekt der elektr. Leitfähigkeit.

Unter der Annahme der exakten Gültigkeit der Theorie der freien Elektronen und völlig, diffuser Reflexion der Elektronen an den inneren Metalloberflächen ergeben sich aus den Meßwerten für Wismut und Blei mittlere freie Weglängen von $2000 \AA$ bei $25^{\circ} \mathrm{C}$ bzw. $84 \AA$ bei $35^{\circ} \mathrm{C}$. Die experimentell gefundene Abnahme der differentiellen Thermokraft stimmt für diese i.-Werte gut mit der theoretischen Kurve überein. Abweichungen bei sehr dünnen Wismutschichten wurden schon in einer früheren Arbeit auf die Bindung der Elektronen und Oberflächeneffekte zurückgeführt.

Im zweiten Teil dieser Arbeit wird auch der Fall teilweise elastischer Reflexion der Elektronen an den inneren Oberflächen des Metalles behandelt. Ferner wird an Hand der allgemeinen Formel die absolute Thermokraft für dünne Drähte berechnet.

Die gefundene Schichtdickenabhängigkeit der Thermokraft ist durch eine rein thermodynamische Betrachtungsweise nicht zu erklären, während auf Grund der Metalltheorie eine zwanglose Deutung als Weglängeneffekt möglich ist. Darüber hinaus tritt aus diesen Betrachtungen die grundsätzliche Bedeutung der auf ein einheitliches Material bezogenen absoluten Thermokraft deutlich hervor.

In einem zusätzlichen Abschnitt wird noch gezeigt, daß die Wärmeleitfähigkeit auch im Falle einer energieabhängigen freien Weglänge mit dem gleichen Korrekturfaktor behaftet wird wie die elektrische Leitfähigkeit, so daß das W i e d e m a nn-F r a n z - L o r e n z sche Gesetz. bei den dünnen Schichten und Drähten keine Modifikation erfährt.

$\mathrm{W}_{\mathrm{m}}$ ird die mittlere freie Weglänge der Elektronen mit den geometrischen Abmessungen eines metallischen Leiters vergleichbar, so kann man im elektrischen und thermischen Verhalten dieser dünnen Metallfilme oder -drähte beträchtliche Anomalien beobachten. In einer vorhergehenden Arbeit hatten wir einen derartigen Weglängeneffekt auf die differentielle Thermokraft dünner Metallschichten experimentell nachgewiesen ${ }^{1}$. Diese Abweichungen von den Eigenschaften des kompakten Materials sind auf Grund einer thermodynamisch-phänomenologischen Theorie der Thermokräfte unverständlich und können nur im Rahmen der Elektronentheorie der Metalle erklärt werden. Letztere führt den gefundenen Effek $\hat{\imath}^{t}$ zum größten Teil auf eine relative Vermehrung der Elektronenstreuprozesse an der Oberfläche im Vergleich zu denen im Innern der Metallschichten zurück

In der vorliegenden Arbeit soll eine Berechnung

1 E. Justi, M. K oh le r u. G. L a u tz, Z. Naturforschg. 6 a, 456 [1951]. der differentiellen Thermokraft dünner metallischer Leiter unter Berücksichtigung des Weglängeneffektes vorgenommen werden.

1. Berechnung der absoluten Thermo$\mathrm{kraft}$ dünner Folien

In einer Metallfolie, deren Länge und Breite groß gegenüber ihrer Dicke sein sollen, möge in der $x$-Richtung ein Temperaturgefälle und ein elektrisches Feld vorhanden sein. Für die Verteilung der Elektronen gilt quantitativ nach der Elektronentheorie der Metalle die Boltzmannsche Fundamentalgleichung

$$
\frac{\partial f}{\partial t}+(\mathfrak{v} \operatorname{grad} f)+\left(\mathfrak{b} \operatorname{grad}_{\mathfrak{v}} f\right)=\left(\frac{\partial f}{\partial t}\right)_{\text {Stöße }} .
$$

Dabei bedeuten $\mathfrak{v}$ die Elektronengeschwindigkeit, $\mathfrak{b}$ deren Beschleunigung und $f$ die gestörte Verteilungsfunktion. Im stationären Falle ist

$$
\frac{\partial f}{\partial t}=0 \text {. }
$$


Unter der Voraussetzung, daß sich die Wechselwirkung der Elektronen mit dem Gitter durch eine einheitliche Relaxationszeit $\tau$ beschreiben läßt, können wir den Lor e n t z schen Ansatz

$$
\left(\frac{\partial f}{\partial t}\right)_{\text {Stöße }}=-\frac{f-f_{0}}{\tau}
$$

einführen, wo $f_{0}$ die ungestörte Fermi-Verteilung ist. Diese Annahme ist im Gebiet tiefer Temperaturen, wo nur der Restwiderstand maßgebend ist, und für $T>\Theta_{\mathrm{D}}\left(\Theta_{\mathrm{D}}=\right.$ Debye sche charakteristische Temperatur) sicher zulässig.

Qrientieren wir das $x, y, z$-System mit der $z$-Achse

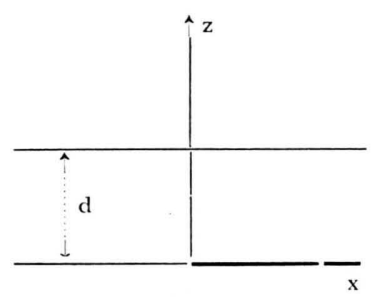

senkrecht zur Schichtebene, so ist $f$ von $y$ nicht abhängig, und man erhält mit

$$
b_{x}=-\frac{e F_{x}}{m}, v_{x} \frac{\partial f}{\partial x}+v_{z} \frac{\partial f}{\partial z}-\frac{e F_{x}}{m} \frac{\partial f}{\partial v_{x}}=-\frac{f-f_{0}}{\tau}
$$

In der üblichen Näherung betrachtet man die Änderung der Verteilungsfunktion durch das elektrische Feld und den Temperaturgradienten in der $x$-Richtung als so klein, daß man auf der linken Gleichungsseite $f$ durch $f_{0}$ ersetzen darf. Das gilt aber nicht mehr bezüglich der $z$-Abhängigkeit, da die Schichtdicke in die Größenordnung der freien Weglänge der Elektronen geraten kann. Mit

$$
f_{1}=f-f_{0}
$$

und $f_{1} \ll f_{0}$ ergibt sich

$$
v_{x} \frac{\partial f_{0}}{\partial x}+v_{z} \frac{\partial f_{1}}{\partial z}-\frac{e F_{x}}{m} \frac{\partial f_{0}}{\partial v_{x}}=-\frac{f_{1}}{\tau} .
$$

Dabei wurde noch angenommen, daß $f_{0}$ eine isotrope Fermi-Verteilung darstellt und damit die Fermische Grenzenergie $\zeta$ von der Schichtdicke nicht abhängt. Wir führen die Elektronenenergie $E$. ein und bekommen nach bekannten Umformungen ${ }^{2}$

$$
v_{z} \frac{\partial f_{1}}{\partial z}+\frac{f_{1}}{\tau}=e v_{x} F_{x} \frac{\partial f_{0}}{\partial E}+v_{x}\left(\frac{\partial \zeta}{\partial x}+\frac{E-\zeta}{T} \frac{\partial T}{\partial x}\right) \frac{\partial f_{0}}{\partial E}
$$

oder mit

$$
F_{x}^{*}=F_{x}+\frac{1}{e} \frac{\partial \zeta}{\partial x}
$$

$$
v_{z} \frac{\partial f_{1}}{\partial z}+\frac{f_{1}}{\tau}=e v_{x} F_{x}^{*} \frac{\partial f_{0}}{\partial E}+v_{x} \frac{E-\zeta}{T} \frac{\partial T}{\partial x} \frac{\partial f_{0}}{\partial E} .
$$

Die Lösung lautet nach $\mathrm{F} \mathrm{u} \mathrm{ch} \mathrm{s}^{3}$

$$
\begin{array}{r}
f_{1}=\left(e v_{x} \tau F_{x}^{*} \frac{\partial f_{0}}{\partial E}+v_{x} \tau \frac{E-\zeta}{T} \frac{\partial T}{\partial x} \frac{\partial f_{0}}{\partial E}\right) \\
\cdot\left(1+\Phi(\mathfrak{y}) e^{-\frac{z}{\tau v_{z}}}\right)
\end{array}
$$

wo $\Phi(\mathfrak{b})$ noch eine willkürliche Funktion von $\mathfrak{v}$ ist, die aus den Randbedingungen bestimmt wird.

Wir wollen die Metalloberfläche als so rauh annehmen, daß die Streuwahrscheinlichkeit eines Elektrons für jeden Raumwinkelbereich zum Innern der Schicht hin gleich groß und unabhängig von der Auftreffrichtung ist. Die Reflexion der Elektronen am Rande der Schicht soll also vollständig diffus sein. Dann gilt nach $\mathrm{F} \mathrm{u} \mathrm{ch} \mathrm{s}{ }^{3}$

$$
\begin{array}{lll}
\Phi(\mathfrak{y})=-1 & \text { für } & v_{z}>0, \\
\Phi(\mathfrak{g})=-e^{\tau v_{z}} & \text { für } & v_{z}<0 .
\end{array}
$$

Zur Berechnung der Thermokraft muß man aus physikalischen Gründen verlangen, daß im offenen Thermokreis der elektrische Gesamtstrom und damit die über den Querschnitt gemittelte elektrische Stromdichte verschwindet. Also folgt mit

$$
\begin{aligned}
& i_{x}=-e \iint v_{x} f_{1} D(E) \mathrm{d} E \mathrm{~d} \Omega, \\
& \overline{i_{x}}=-\frac{e}{d} \int_{0}^{d} \mathrm{~d} z \iint v_{x} f_{1} D(E) \mathrm{d} E \mathrm{~d} \Omega=0,
\end{aligned}
$$

wobei wie üblich

$$
D(E) \mathrm{d} E=\left(\frac{2 m}{h^{2}}\right)^{3 / 2} E^{1 / 2} \mathrm{~d} E
$$

die Anzahl von Elektronenzuständen innerhalb $E$... $E+\mathrm{d} E$ und in der Raumwinkeleinheit des Geschwindigkeitsraumes bedeuten. Zur Vereinfachung der Rechnung führen wir in letzterem Polarkoordinaten ein

$$
v_{z}=v \cos \vartheta, \quad v_{x}=v \sin \vartheta \cos \varphi
$$

$2 \mathrm{H}$. Fröhlich, Elektronentheorie der Metalle, Springer, Berlin 1936.

3 K. F u ch s, Proc. Cambridge philos. Soc. 34, 100 [1938]. 
und erhalten

$$
\begin{aligned}
\overline{i_{x}}=0= & -\frac{e}{d} \int_{E=0}^{\infty} \int_{\vartheta=0}^{\pi / 2} \int_{\varphi=0}^{2 \pi} e F_{x}^{*} \tau \frac{\partial f_{0}}{\partial E} v^{4} \sin ^{3} \vartheta \cos ^{2} \varphi \int_{0}^{d}\left(1-e^{\left.-\frac{z}{v \tau \cos \vartheta}\right)} \mathrm{d} z D(E) \mathrm{d} E \mathrm{~d} \vartheta \mathrm{d} \varphi\right. \\
& -\frac{e}{d} \int_{E=0}^{\infty} \int_{\vartheta=\pi / 2}^{\pi} \int_{\varphi=0}^{2 \pi} e F_{x}^{*} \tau \frac{\partial f_{0}}{\partial E} v^{4} \sin ^{3} \vartheta \cos ^{2} \varphi \int_{0}^{d}\left(1-e^{-\frac{z-d}{v \tau \cos \vartheta}}\right) \mathrm{d} z D(E) \mathrm{d} E \mathrm{~d} \vartheta \mathrm{d} \varphi \\
& -\frac{e}{d} \int_{E=0}^{\infty} \int_{\vartheta=0}^{\pi / 2} \int_{\varphi=0}^{2 \pi} \frac{E-\zeta}{T} \frac{\partial T}{\partial x} \tau \frac{\partial f_{0}}{\partial E} v^{4} \sin ^{3} \vartheta \cos ^{2} \varphi \int_{0}^{d}\left(1-e^{-\frac{z}{\tau v \cos \vartheta}}\right) \mathrm{d} z D(E) \mathrm{d} E \mathrm{~d} \vartheta \mathrm{d} \varphi \\
& -\frac{e}{d} \int_{E=0}^{\infty} \int_{\vartheta=\pi / 2}^{\pi} \int_{\varphi=0}^{2 \pi} \frac{E-\zeta}{T} \frac{\partial T}{\partial x} \tau \frac{\partial f_{0}}{\partial E} v^{4} \sin ^{3} \vartheta \cos ^{2} \varphi \int_{0}^{d}\left(1-e^{-\frac{z-d}{v \tau \cos \vartheta}}\right) \mathrm{d} z D(E) \mathrm{d} E \mathrm{~d} \vartheta \mathrm{d} \varphi .
\end{aligned}
$$

Zur Abkürzung setzen wir

$$
\psi(E) \equiv \frac{1}{d} \int_{0}^{d} \mathrm{~d} z \int_{0}^{\pi / 2} \sin ^{3} \vartheta\left(1-e^{-\frac{z}{\tau v \cos \vartheta}}\right) \mathrm{d} \vartheta+\frac{1}{d} \int_{0}^{d} \mathrm{~d} z \int_{\pi / 2}^{\pi} \sin ^{3} \vartheta\left(1-e^{-\frac{z-d}{v \tau \cos \vartheta}}\right) \mathrm{d} \vartheta .
$$

Damit folgt aus (9)

$$
\overline{i_{x}}=0=-\frac{2 \pi e^{2}}{m} F_{x}^{*} \int_{0}^{\infty} \tau E D(E) \psi(E) \frac{\partial f_{0}}{\partial E} \mathrm{~d} E-\frac{2 \pi e}{m T} \frac{\partial T}{\partial x} \int_{0}^{\infty} \tau E(E-\zeta) D(E) \psi(E) \frac{\partial f_{0}}{\partial E} \mathrm{~d} E .
$$

Führen wir weiter als Abkürzung einen der elektrischen Leitfähigkeit ähnlichen Ausdruck

$$
\sigma(E)=\frac{2 \pi e^{2}}{m} \tau E D(E) \psi(E)
$$

ein, so ergibt sich

$$
\begin{aligned}
\overline{i_{x}}=0 & =-F_{x}^{*} \int_{0}^{\infty} \sigma(E) \frac{\partial f_{0}}{\partial E} \mathrm{~d} E \\
& \quad-\frac{1}{e T} \frac{\partial T}{\partial z} \int_{0}^{\infty} \sigma(E)(E-\zeta) \frac{\partial f_{0}}{\partial E} \mathrm{~d} E .
\end{aligned}
$$

Zur Berechnung der thermoelektrischen Feldstärke $F_{x}{ }^{*}$ genügt es, bei der Auswertung der Sommer$\mathrm{f}$ eldschen Integrale im ersten Integral die erste Näherung zu nehmen. Man erhält

$$
\sigma=F_{x}^{*} \sigma(\xi)+\frac{1}{e T} \frac{\partial T}{\partial x} \frac{\pi^{2}}{3}(k T)^{2} \sigma^{\prime}(\zeta),
$$

wo $\sigma(\zeta)$ die von $\mathrm{Fu} \mathrm{chs}^{3}$ für die dünne Schicht berechnete elektrische Leitfähigkeit ist. Also gilt für die thermoelektrische Feldstärke

$$
F_{x}^{*}=-\frac{1}{e} \frac{\pi^{2}}{3} k^{2} T \frac{\partial T}{\partial x}\left(\frac{\mathrm{d} \log \sigma}{\mathrm{d} E}\right)_{E=\xi} .
$$

Nun wird in der Elektronentheorie der Metalle ge- zeigt, daß der Faktor von $\partial T / \partial x$ in diesem Ausdruck gleich der absoluten Thermokraft $\varepsilon$ ist ${ }^{2}$.

$$
\varepsilon=-\frac{1}{e} \frac{\pi^{2}}{3} k^{2} T\left(\frac{\mathrm{d} \log \sigma}{\mathrm{d} E}\right)_{E=\zeta} .
$$

Unter Benutzung des Ausdrucks für $\sigma$ folgt sofort

$$
\frac{\mathrm{d} \log \sigma}{\mathrm{d} E}=\frac{\mathrm{d} \log (\tau D(E))}{\mathrm{d} E}+\frac{\mathrm{d} \log \psi(E)}{\mathrm{d} E} .
$$

Das erste Glied ist vom kompakten Material her bekannt, das zweite Glied liefert den Einfluß der Schichtdicke auf die absolute Thermokraft. Setzen wir deren Abweichung vom Wert des kompakten Materials gleich $\Delta \varepsilon$, so wird

$$
\lrcorner \varepsilon=-\frac{1}{e} \frac{\pi^{2}}{3} k^{2} T\left(\frac{\mathrm{d} \log \psi(E)}{\mathrm{d} E}\right)_{E=\zeta} .
$$

Zur weiteren Auswertung dieser Formel benutzen wir das von Fuchs berechnete Verhältnis der elektrischen Leitfähigkeit einer dünnen Schicht zu der des kompakten Materials

$$
\begin{aligned}
\psi(E)= & \frac{\sigma}{\sigma_{\infty}}=1-\frac{3}{8 \%}\left(1-e^{-\varkappa}\right) \\
& +\frac{3}{4}\left(\varkappa-\frac{\varkappa^{3}}{12}\right) B(\%)-\left(\frac{5}{8}+\frac{\varkappa}{16}-\frac{\varkappa^{2}}{16}\right) e^{-\varkappa}
\end{aligned}
$$


mit

$$
B(\varkappa)=\int_{\varkappa}^{\infty} \frac{e^{-x}}{x} \mathrm{~d} x
$$

wo

$$
\% \equiv \frac{d}{\lambda}=\frac{d}{\tau v}
$$

mit $\lambda$ als mittlerer freier Weglänge. Da $\psi$ nur mittelbar über die freie Weglänge von der Energie ab- hängen kann, gilt

$$
\frac{\mathrm{d} \log \psi}{\mathrm{d} E}=\frac{\mathrm{d} \log \psi}{\mathrm{d} \varkappa} \frac{\mathrm{d} \varkappa}{\mathrm{d} \lambda} \frac{\mathrm{d} \lambda}{\mathrm{d} E}=-\frac{\mathrm{d} \log \lambda}{\mathrm{d} E} \varkappa \frac{\mathrm{d} \log \psi}{\mathrm{d} \varkappa} .
$$

Damit ist sofort evident, daß $\Delta \varepsilon$ für eine energieunabhängige freie Weglänge der Elektronen verschwindet und kein Schichtdickeneffekt bei der Thermokraft auftreten kann. Umgekehrt dürfen wir aus dem Vorhandensein des Schichtdickeneffektes schließen, daß $\lambda$ eine Funktion der Energie sein muß.

Die Ausrechnung liefert

und damit

$$
\frac{\mathrm{d}\left(\sigma / \sigma_{\infty}\right)}{\mathrm{d} \varkappa}=\frac{3}{8 \varkappa^{2}}\left(1-e^{-\varkappa}\right)+\frac{3}{4}\left(1-\frac{\varkappa^{2}}{4}\right) B(\varkappa)+\left(-\frac{3}{16}-\frac{3}{8 \%}+\frac{3 \varkappa}{16}\right) e^{-\varkappa}
$$

$$
\Delta \varepsilon=-\frac{1}{e} \frac{\pi^{2}}{3} \frac{k^{2} T}{\zeta}\left(\frac{\mathrm{d} \log \lambda}{\mathrm{d} \log E}\right)_{E=\zeta} \frac{-\frac{3}{8 \varkappa}\left(1-e^{-\varkappa}\right)-\frac{3}{4}\left(\varkappa-\frac{\varkappa^{3}}{4}\right) B(\varkappa)+\left(\frac{3}{8}+\frac{3}{16} \varkappa-\frac{3}{16} \varkappa^{2}\right) e^{-\varkappa}}{1-\frac{3}{8 \varkappa}\left(1-e^{-\varkappa}\right)+\frac{3}{4}\left(\varkappa-\frac{\varkappa^{3}}{12}\right) B(\varkappa)-\left(\frac{5}{8}+\frac{\varkappa}{16} \frac{\varkappa^{2}}{16}\right) e^{-\varkappa}} .
$$

Die absolute Thermokraft einer dünnen Schicht wird also mit

$$
\varepsilon_{\infty}=-\frac{1}{e} \frac{\pi^{2}}{3} \frac{k^{2} T}{\zeta}\left[1+\left(\frac{\mathrm{d} \log \lambda}{\mathrm{d} \log E}\right)_{E=\zeta}\right]
$$

$\varepsilon=\varepsilon_{\infty}+\Delta \varepsilon=-\frac{1}{e} \frac{\pi^{2}}{3} \frac{k^{2} T}{\zeta}\left[1+\left(\frac{\mathrm{d} \log \lambda}{\mathrm{d} \log E}\right)_{E=\zeta} \frac{1-\frac{3}{4 \varkappa}\left(1-e^{-\varkappa}\right)+\frac{\varkappa^{3}}{8} B(\varkappa)-\left(\frac{1}{4}-\frac{\varkappa}{8}+\frac{\varkappa^{2}}{8}\right) e^{-\varkappa}}{1-\frac{3}{8 \varkappa}\left(1-e^{-\varkappa}\right)+\frac{3}{4}\left(\varkappa-\frac{\varkappa^{3}}{12}\right) B(\varkappa)-\left(\frac{5}{8}+\frac{\varkappa}{16}-\frac{\varkappa^{2}}{16}\right) e^{-\varkappa}}\right]$.

Für große $\varkappa$, d. h. dicke Schichten, gilt

$$
\lim _{x \gg 1} \varepsilon=-\frac{1}{e} \frac{\pi^{2}}{3} \frac{k^{2} T}{\zeta}\left[1+\left(\frac{\mathrm{d} \log \lambda}{\mathrm{d} \log E}\right)_{E=\zeta}\left(1-\frac{3}{8 \%}\right)\right] .
$$

Als erste Korrektur an dem Wert des kompakten Materials erhält man so ein lineares Glied, wie man es auch von der elektrischen Leitfähigkeit her kennt. F'ür kleine $\varkappa$, d. h. sehr dünne Schichten, gilt

$$
B(\varkappa) \rightarrow \log \frac{1}{\gamma \varkappa},
$$

wo $\gamma$ ein Zahlenfaktor ist. Damit folgt

$$
\lim _{x \rightarrow 0} \varepsilon=-\frac{1}{e} \frac{\pi^{2}}{3} \frac{k^{2} T}{\zeta} .
$$

2. Berücksichtigung einer teilweise elastischen Reflexion der Elektronen an der Leiteroberfläche

Wir haben bisher stets den Fall vollständig diffuser Reflexion der Elektronen an den inneren Metalloberflächen angenommen. Es kann aber nicht ausgeschlossen werden, daß ein Teil dieser Reflexionen elastisch verläuft, wenngleich deren $\mathrm{Zahl}$ in Wirklichkeit stets kleiner als die der unelastischen Streuungen bleiben dürfte.

Ist $q$ die Wahrscheinlichkeit dafür, daß ein Elektron elastisch an der Oberfläche reflektiert wird, so gilt nach $\mathrm{Fuchs}{ }^{3}$

$\frac{\sigma}{\sigma_{\infty}}=1-\frac{3(1-q)}{8 \%}+\frac{3(1-q)^{2}}{4 \varkappa} \sum_{\nu=1}^{\infty} q^{\nu-1}\left\{B(\varkappa v)\left(\varkappa^{2} v^{2}-\frac{\varkappa^{4} v^{4}}{12}\right)+e^{-\varkappa v}\left(\frac{1}{2}-\frac{5}{6} \varkappa v-\frac{\varkappa^{2} v^{2}}{12}+\frac{\varkappa^{3} v^{3}}{12}\right)\right\}$. 
Zur Auswertung für die absolute Thermokraft benötigen wir nach (14) den Ausdruck $\varkappa \frac{\mathrm{d}\left(\sigma / \sigma_{\infty}\right)}{\mathrm{d} \varkappa}=\frac{3(1-q)}{8 \%}-\frac{3(1-q)^{2}}{4 \%} \sum_{v=1}^{\infty} q^{\nu-1}\left\{B(\varkappa v)\left(-\varkappa^{2} v^{2}+\frac{\varkappa^{4} v^{4}}{4}\right)+e^{-\varkappa v}\left(\frac{1}{2}+\frac{\varkappa v}{2}+\frac{\varkappa^{2} v^{2}}{4}-\frac{\varkappa^{3} v^{3}}{4}\right)\right\}$.

Daraus folgt mit (14)

$$
\begin{aligned}
\Delta \varepsilon=- & \frac{1}{e} \frac{\pi^{2}}{3} \frac{k^{2} T}{\zeta}\left(\frac{\mathrm{d} \log \lambda}{\mathrm{d} \log E}\right)_{E=\xi} \\
& -\frac{3(1-q)}{8 \%}+\frac{3(1-q)^{2}}{4 \%} \sum_{v=1}^{\infty} q^{v-1}\left\{B(\varkappa v)\left(-\varkappa^{2} v^{2}+\frac{\varkappa^{4} v^{4}}{4}\right)+e^{--\varkappa v}\left(\frac{1}{2}+\frac{\varkappa v}{2}+\frac{\varkappa^{2} v^{2}}{4}-\frac{\varkappa^{5} v^{3}}{4}\right)\right\} \\
& \cdot \frac{3(1-q)}{8 \%}+\frac{3(1-q)^{2}}{4 \%} \sum_{v=1}^{\infty} q^{v-1}\left\{B(\varkappa v)\left(\varkappa^{2} v^{2}-\frac{\varkappa^{4} v^{4}}{12}\right)+e^{-\varkappa v}\left(\frac{1}{2}-\frac{5}{6} \% v-\frac{\varkappa^{2} v^{2}}{12}+\frac{\varkappa^{3} v^{3}}{12}\right)\right\}
\end{aligned}
$$

und

$\varepsilon=-\frac{1}{e} \frac{\pi^{2}}{3} \frac{k^{2} T}{\zeta}$

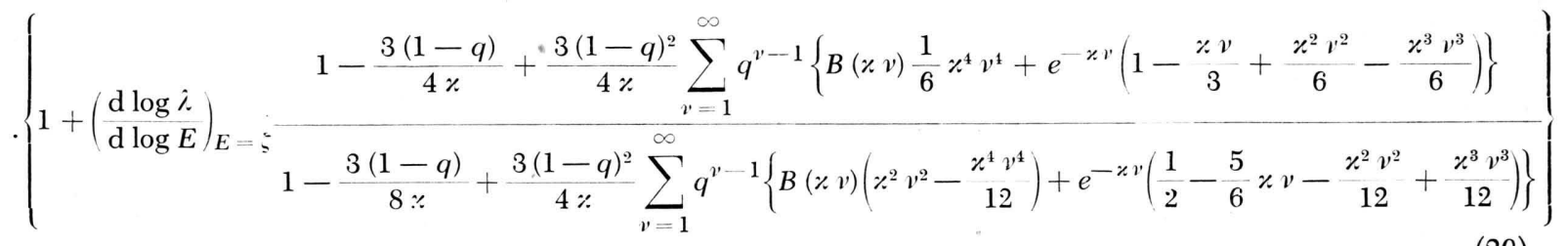

Im Grenzfall vollständig elastischer Reflexion verschwindet der Schichtdickeneffekt, da die Streuprozesse an der Leiteroberfläche keine Änderung der elektrischen oder thermischen Stromdichte bedingen. Mit abnehmendem $q$ wird der Weglängeneffekt größer und erreicht für $q=0$ den bereits in (17) berechneten Wert.

Für sehr dicke Schichten erhält man $(x \geqslant 1)$

$$
\begin{aligned}
\lim _{x \gg 1} \varepsilon= & -\frac{1}{e} \frac{\pi^{2}}{3} \frac{k^{2} T}{\zeta} \\
& \cdot\left\{1+\left(\frac{\mathrm{d} \log \lambda}{\mathrm{d} \log E}\right)_{E=\{}\left[1-\frac{3}{8 \varkappa}(1-q)\right]\right\} .
\end{aligned}
$$

(20a)
3. Berechnung der absoluten Thermokraft dünner Drähte

Ganz analog zu den Berechnungen für die dünnen Schichten lassen sich auch die Formeln für die dünnen Drähte finden. Zu diesem Zweck braucht man nur die von $\mathrm{D}$ ingle ${ }^{4}$ angegebene elektrische Leitfähigkeit in (14) einzusetzen und erhält mit

$$
\frac{\sigma}{\sigma_{\infty}}=1-\frac{12}{\pi} \int_{0}^{1}\left(1-\xi^{2}\right)^{1 / 2} S_{4}(x \xi) \mathrm{d} \xi
$$

$$
\begin{gathered}
\varkappa \frac{\mathrm{d}\left(\sigma / \sigma_{\infty}\right)}{\mathrm{d} \varkappa}=-\frac{12}{\pi} * \int_{0}^{1} \xi\left(1-\xi^{2}\right)^{1 / 2} S_{4}{ }^{\prime}(\% \xi) \mathrm{d} \xi \\
\varepsilon=-\frac{1}{e} \frac{\pi^{2}}{3} \frac{k^{2} T}{\zeta}\left\{1+\left(\frac{\mathrm{d} \log \lambda}{\mathrm{d} \log E}\right)_{E=\xi} \frac{1+\frac{12}{\pi} \int_{0}^{1}\left(1-\xi^{2}\right)^{1 / 2}\left[S_{4}{ }^{\prime}(\% \xi) \xi-S_{4}(\% \xi)\right] \mathrm{d} \xi}{1-\frac{12}{\pi} \int_{0}^{1}\left(1-\xi^{2}\right)^{1 / 2} S_{4}(\varkappa \xi) \mathrm{d} \xi}\right\} .
\end{gathered}
$$

4 R. B. D ingle, Proc. Roy. Soc. [London], Ser. A 202, 545 [1950]. 
Dabei kann die numerische Auswertung der Integrale nach dem von Dingle angegebenen Näherungsverfahren erfolgen.

Praktisch interessiert nur der Fall dicker Drähte, also $x \gg 1$. Die entsprechende Näherungsformel von D ing le ${ }^{4}$ lautet

$$
\lim _{x \gg 1} \frac{\sigma}{\sigma_{\infty}}=1-\frac{3}{4 \%} .
$$

Also folgt für die Thermokraft

$\lim _{* \gg 1} \varepsilon=-\frac{1}{e} \frac{\pi^{2}}{3} \frac{k^{2} T}{\zeta}\left\{1+\left(\frac{\mathrm{d} \log \lambda}{\mathrm{d} \log E}\right)_{E=\xi}\left(1-\frac{3}{4 \%}\right)\right\}$.

Auf eine gesonderte Diskussion einer teilweise elastischen Reflexion der Elektronenwellen für den Fall der dünnen Drähte sei hier verzichtet, da sich hierbei nichts grundsätzlich Neues ergibt.

4. Diskussion der Ergebnisse und ein Vergleich mit Experimenten an Pbund Bi-Schichten

Die Abb. 1 zeigt die auf das kompakte Material bezogene prozentuale Abnahme der absoluten Thermokraft als Funktion des Verhältnisses der freien Weglänge zur Schichtdicke nach Gl. (20) mit zum Vergleich eingetragenen Meßpunkten.

Dabei wurde außerdem $\lambda=$ const. $E^{n}$ gesetzt; die gemäß $\left(\frac{\mathrm{d} \log \lambda}{\mathrm{d} \log E}\right)_{E=\xi}$ verschieden starke Abnahme ist für $n=1,2$ und 4 dargestellt. $n=2$ würde der B loch schen Theorie entsprechen.

Durch die sehr weitgehende Kompensation zweier Glieder in dem Ausdruck für $\varepsilon$ gilt die lineare Näherung bis etwa $\lambda / d=2 / 3$. In diesem Gebiet kann man aus den Meßwerten die freie Weglänge berechnen, wenn eine plausible Annahme über $n$ möglich ist. Hat man aber die Weglänge bereits aus anderen Messungen, so liefert die Schichtdickenabhängigkeit der absoluten Thermokraft die Energieabhängigkeit de: freien Weglänge. Derartige Messungen ermöglichen also einen viel tieferen Einblick in den Leitungsmechanismus als die Leitfähigkeitsmessungen allein, die gegenüber einer Änderung des Stoßmechanismus ziemlich unempfindlich sind.

Die in der Abb. 1 eingezeichneten Meßpunkte an dünnen $\mathrm{Pb}$ - und $\mathrm{Bi}$-Schichten sind der Kurve für $n=2$ durch eine geeignete Wahl von $\lambda$ möglichst gut angepaßt worden. Die ursprünglich gegen Kupfer gemessenen differentiellen Thermokräfte wurden unter Benutzung der absoluten Thermokräfte des
Kupfers $\left(1,93 \mu \mathrm{V} / \mathrm{Grad}\right.$ bei $\left.35^{\circ} \mathrm{C}\right)$ nach B or eliu s ${ }^{5}$ und $\mathrm{N}$ y $\mathrm{t}$ r ö $\mathrm{m}^{6}$ ihrerseits auf die Absolutwerte umgerechnet.

Für Wismut ergab sich unter der Voraussetzung der strengen Gültigkeit dieser Theorie eine Weglänge von $2000 \AA$ bei $25^{\circ} \mathrm{C}$. Die den größeren $\lambda / d$-Werten entsprechenden Meßpunkte weichen erheblich von der theoretischen Kurve ab. Das ist im Falle des Wismut nicht so verwunderlich, da bei diesem Metall die Näherung freier Elektronen sicher nur eine sehr rohe Methode darstellt. Auf einige Deutungsmöglichkeiten des großen Schichtdickeneffektes haben wir bereits hingewiesen ${ }^{1}$.

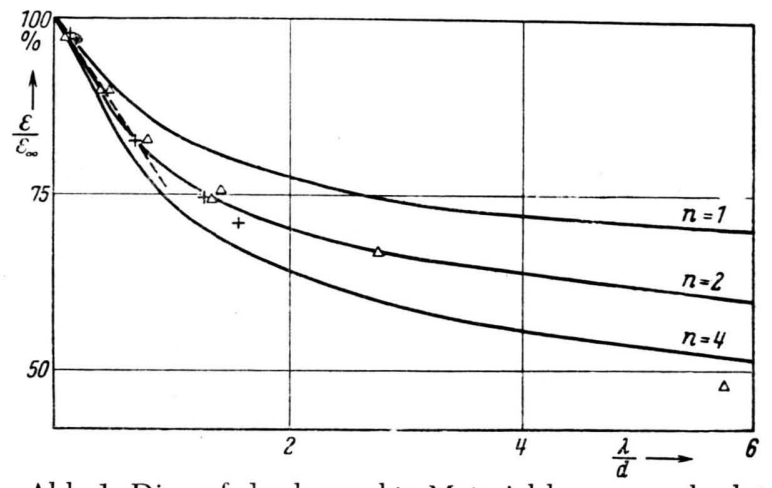

Abb. 1. Die auf das kompakte Material bezogene absolute Thermokraft dünner Metallschichten als Funktion von $\varkappa=\lambda / d$ für verschiedene Exponenten $n\left(\lambda=\right.$ const. $\left.E^{n}\right)$.

$+\mathrm{Pb}$-Schichten bei $35^{\circ} \mathrm{C} ; \triangle \mathrm{Bi}$-Schichten bei $25^{\circ} \mathrm{C}$.

Die Voraussetzungen der Theorie sind beim Blei wesentlich besser erfüllt. Die ebenfalls für $n=2$ und völlig diffuse Reflexion eingezeichneten Meßpunkte ergeben eine mittlere freie Weglänge von $84 \AA$ bei $35^{\circ} \mathrm{C}$. Dieser Wert stimmt mit Messungen des Weglängeneffektes der elektrischen Leitfähigkeit von $\mathrm{Riedel^{7 }}$ befriedigend überein. Wertet man die Riedelschen Ergebnisse nach der Fuchsschen Theorie aus, so erhält man bei $35^{\circ} \mathrm{C}$ eine freie Weglänge von etwa $61 \AA$. Da nun die Riedelschen Meßdrähte annähernd 1000-mal dicker waren als die von uns untersuchten dünnen Schichten (Riedel untersuchte den Weglängeneffekt bei Wasserstofftemperaturen), könnte ein Teil der Elektronen elastisch reflektiert worden sein, während ein solcher Effekt bei unseren Bleischichten ziemlich ausgeschlossen ist. Nimmt man an, daß nur jedes dritte Elektron bei den Riedelschen

5 G. B o r e li u s, Handbuch der Metallphysik, herausgeg. von G. Masing, Akad. Verl.-Ges. Leipzig 1935.

6 J. Nyström, Ark. Mat., Astronom. Fysik, Ser. A 34, No. 27 [1947].

7 L. Ri e de l, Ann. Physik (5) 28, 603 [1937]. 
Messungen elastisch gestreut worden ist, kommt main zu einer vollständigen Übereinstimmung beider Angaben über die mittlere freie Weglänge. Aus dem $\lambda$-Wert von $84 \AA$ erhält man etwa 0,7 freie Elektronen je Atom, ein Wert, der auch mit anderen Abschätzungen von $\mathrm{Mott}^{8}$ und $\mathrm{Fröhlich}{ }^{2}$ befriedigend übereinstimmt.

Unser zur Herstellung der Aufdampfschichten benutztes Blei zeigte einen Restwiderstand von $4,5 \cdot 10^{-4}$, bezogen auf den Eispunktswiderstand, und liegt damit nur wenig höher als der beste Literaturwert ${ }^{9}$ von $1,55 \cdot 10^{-4}$. Bezüglich der experimentellen Methodik verweisen wir auf unsere vorhergehende Arbeit ${ }^{1}$. Infolge des außerordentlich kleinen Effektes beim Blei ist es uns nicht gelungen, für $\lambda / d>2$ einwandfreie Messungen auszuführen. Da man hier bald in die Größenordnung der Kristallite kommt, würde auch die Gültigkeit der vorliegenden Theorie für diesen Bereich sehr in Frage gestellt.

5. Eine Bemerkung zur Wärmeleitfähigkeit dünner Schichten

In der Arbeit von Dingle über die elektrische Leitfähigkeit dünner Drähte findet sich ein Hinweis auf die Gültigkeit des Wiedemann-Franz-Lorenzschen Gesetzes, nach dem das Verhältnis der thermischeiı zur elektrischen Leitfähigkeit bei konstanter Temperatur eine Konstante sein soll:

$$
\frac{\sigma_{\mathrm{Th}}}{\sigma T}=L
$$

( $\sigma_{\mathrm{Th}}=$ thermische Leitfähigkeit, $L=$ Lorenzsche Zahl).

Diese Bemerkung bezieht sich auf den Fall einer energieunabhängigen mittleren freien Weglänge. Es ist aber leicht einzusehen, daß die thermische Leitfähigkeit dünner Drähte und Filme auch bei einer Energieabhängigkeit des $\lambda$ mit dem gleichen Korrekturfaktor $\mathrm{zu}$ versehen ist wie die elektrische Leitfähigkeit.

Definitionsgemäß ist die Wärmestromdichte durch

$$
Q_{x}=\iint E v_{x} f_{1} D(E) \mathrm{d} E \mathrm{~d} \Omega
$$

gegeben, die analog zur elektrischen Stromdichte

$$
i_{x}=-e \iint v_{x} f_{1} D(E) \mathrm{d} E \mathrm{~d} Q
$$

8 N. F. M ot t, Proc. physic. Soc. (A) 147, 680 [1934]. 9 W. Meißner, Ann. Physik (5) 13, 641 [1932]. mit dem in (4) berechneten $f_{1}$ zu bilden ist. Nach dem Einsetzen von (4) in (6) und (24) ergibt sich unter Berücksichtigung der Abkürzung $\psi(E)$ aus (10):

$$
\begin{aligned}
\overline{Q_{x}} & =\frac{2 \pi e}{m} F_{x}^{*} \int_{0}^{\infty} \tau E^{2} D(E) \psi(E) \frac{\partial f_{0}}{\partial E} \mathrm{~d} E \\
& +\frac{2 \pi}{m T} \frac{\partial T}{\partial x} \int_{0}^{\infty} \tau E^{2}(E-\zeta) D(E) \psi(E) \frac{\partial f_{0}}{\partial E} \mathrm{~d} E . \\
\overline{j_{x}} & =-\frac{2 \pi e^{2}}{m} F_{x}^{*} \int_{0}^{\infty} \tau E D(E) \psi(E) \frac{\partial f_{0}}{\partial E} \mathrm{~d} E \\
& -\frac{2 \pi e}{m T} \frac{\partial T}{\partial x} \int_{0}^{\infty} \tau E(E-\zeta) D(E) \psi(E) \frac{\partial f_{0}}{\partial E} \mathrm{~d} E .
\end{aligned}
$$

In diesen beiden Ausdrücken kann man nun formal dem Schichtdickeneffekt dadurch Rechnung tragen, daß man $\tau \psi(E)$ zu einer Größe $\tau^{*}$ vereinigt. Dann stimmen (25) und (9a) mit den vom kompakten Material her bekannten Formeln überein, nur daß $\tau(E)$ durch $\tau^{*}(E)$ zu ersetzen ist. Der Weglängeneffekt wirkt sich formal in einer Änderung der Relaxationszeit aus.

Man kann daher mit dieser Modifikation eines veränderten $\tau$ die aus der gewöhnlichen Elektronentheorie her bekannten Formeln für die dünnen Schichten und Drähte übernehmen. Das bedeutet aber, daß das Wiedemann-Franzsche Gesetz in aller Strenge gültig ist, da seine Ableitung in der Elektronentheorie der Metalle von irgendwelchen Annahmen über die Energieabhängigkeit der Relaxationszeit unberührt bleibt. Also folgt aus (23) in Erweiterung der Bemerkung von Dingle, daß die thermische Leitfähigkeit auch im Falle einer energieabhängigen mittleren freien Weglänge ebenso wie die elektrische Leitfähigkeit für die dünne Schicht durch

$$
\begin{aligned}
& \frac{\sigma_{\mathrm{Th}}}{\sigma_{\mathrm{Th}_{\infty}}}=1-\frac{3}{8 \varkappa}\left(1-e^{-\varkappa}\right) \\
& +\frac{3}{4}\left(\varkappa-\frac{\varkappa^{3}}{12}\right) B(\varkappa)-\left(\frac{5}{8}+\frac{\varkappa}{16}-\frac{\varkappa^{2}}{16}\right) e^{-\varkappa}
\end{aligned}
$$

und für den dünnen Draht durch

$$
\frac{\sigma_{\mathrm{Th}}}{\sigma_{\mathrm{Th}_{\infty}}}=1-\frac{12}{\pi} \int_{0}^{1}\left(1-\xi^{2}\right)^{1 / 2} S_{4}(\varkappa \xi) \mathrm{d} \xi
$$

gegeben ist. 